\title{
Clinical outcomes after long-term treatment with alglucosidase alfa in infants and children with advanced Pompe disease
}

\author{
Marc Nicolino, $M D, P h D^{1}$, Barry Byrne, MD, PhD' ${ }^{2}$ J. Edmund Wraith, MD ${ }^{3}$, Nancy Leslie, MD ${ }^{4}$, \\ Hanna Mandel, $M D^{5}$, David R. Freyer, DO ${ }^{6}$, Georgianne L. Arnold, MD ${ }^{7}$, Eniko K. Pivnick, $M D^{8}$, \\ C.J. Ottinger, $M D^{9}$, Peter H. Robinson, $M D^{10}$, John-Charles A. Loo, MD ${ }^{11}$, Martin Smitka, $M D^{12}$, \\ Philip Jardine, $M D^{13}$, Luciano Tatò, $M D^{14}$, Brigitte Chabrol, $M D^{15}$, Shawn McCandless, $M D^{16}$, \\ Shigemi Kimura, $M D^{17}$, L. Mehta, $M D^{18}$, Deeksha Bali, $P h D^{19}$, Alison Skrinar, MA, MPH ${ }^{20}$, \\ Claire Morgan, $M D, M P H^{20}$, Lakshmi Rangachari, $P h D^{20}$, Deya Corzo, $M D^{21}$, \\ and Priya S. Kishnani, $M D^{19}$
}

\begin{abstract}
Purpose: A clinical trial was conducted to evaluate the safety and efficacy of alglucosidase alfa in infants and children with advanced Pompe disease. Methods: Open-label, multicenter study of IV alglucosidase alfa treatment in 21 infants 3-43 months old (median 13 months) with minimal acid $\alpha$-glucosidase activity and abnormal left ventricular mass index by echocardiography. Patients received IV alglucosidase alfa every 2 weeks for up to 168 weeks (median 120 weeks). Survival results were compared with an untreated reference cohort. Results: At study end, 71\% (15/21) of patients were alive and $44 \%$ (7/16) of invasive-ventilator free patients remained so. Compared with the untreated reference cohort, alglucosidase alfa reduced the risk of death by $79 \%(P<0.001)$ and the risk of invasive ventilation by $58 \%(P=0.02)$. Left ventricular mass index improved or remained normal in all patients evaluated beyond 12 weeks; $62 \%$ (13/21) achieved new motor milestones. Five patients were walking independently at the end of the study and $86 \%(18 / 21)$ gained functional independence skills. Overall, $52 \%(11 / 21)$ of patients experienced infusion-associated reactions; $95 \%$ (19/20) developed IgG antibodies to recombinant human lysosomal acid $\alpha$-glucosidase; no patients withdrew from the study because of safety concerns. Conclusions: In this population of infants with advanced disease, biweekly infusions with alglucosidase alfa prolonged survival and invasive ventilation-free survival. Treatment also improved indices of cardiomyopathy, motor skills, and functional independence. Genet Med 2009:11(3):210-219.
\end{abstract}

Key Words: Pompe disease, glycogen storage disease type II, acid maltase deficiency, Myozyme, alglucosidase alfa, lysosomal acid $\alpha$-glucosidase, recombinant human GAA, enzyme replacement therapy, cardiomyopathy, motor development

$P_{\mathrm{f}}$ ompe disease is an autosomal-recessive disorder resulting from a lysosomal acid $\alpha$-glucosidase (GAA) deficiency. The disease causes lysosomal glycogen to accumulate in various

From the ${ }^{1}$ Division of Pediatric Endocrinology, Diabetology and Metabolism, Hôpital Debrousse, University Lyon, Lyon, France; ${ }^{2}$ Shands Hospital, University of Florida, Gainesville, Florida; ${ }^{3}$ Royal Manchester Children's Hospital, Manchester, United Kingdom; ${ }^{4}$ Cincinnati Children's Hospital Medical Center, Cincinnati, Ohio; ${ }^{5}$ Rambam Medical Center, Haifa, Israel; ${ }^{6}$ DeVos Children's Hospital, Grand Rapids, Michigan; ${ }^{7}$ University of Rochester Medical Center, Rochester, New York; ${ }^{8}$ University of Tennessee Health Science Center, Memphis, Tennessee; ${ }^{9}$ Fort Wayne Neurological Center, Fort Wayne, Indiana; ${ }^{10}$ Royal Hospital for Sick Children, Edinburgh, United Kingdom; ${ }^{11}$ Long Beach Memorial Medical Center, Long Beach, California; ${ }^{12}$ Technical University, Dresden, Germany; ${ }^{13}$ Bristol Royal Hospital for Children, Bristol, United Kingdom; ${ }^{14}$ University of Verona, Verona, Italy; ${ }^{15}$ Hopital Timone Enfants, Marseille, France; ${ }^{16}$ University Hospitals of Cleveland, Cleveland, Ohio; ${ }^{17}$ Kumamoto University Graduate School, Kumamoto, Japan; ${ }^{18}$ Mount Sinai Medical Center, New York, New York; ${ }^{19}$ Duke University Medical Center, Durham, North Carolina; ${ }^{20}$ Genzyme Corporation, Cambridge, MA; and ${ }^{21}$ Millennium Pharmaceuticals Inc., Cambridge, MA tissues, particularly muscle, resulting in progressive muscle dysfunction. ${ }^{1}$ The rate of disease progression is, in general, inversely related to the amount of GAA activity in fibroblasts. Activity below detection limits leads to rapid accumulation of glycogen in the muscle, resulting in a rapidly progressive disease and early death, whereas patients with detectable low GAA activity tend to experience a slower but relentlessly progressing disease. Regardless of the level of GAA activity, Pompe disease is associated with high morbidity and mortality. ${ }^{2}$

For decades, treatment for all forms of Pompe disease consisted of supportive care to alleviate symptoms. In 2006, alglucosidase alfa, an enzyme replacement therapy (ERT) that specifically targets the underlying cause of symptoms was approved for commercial use in North America and the EU. Alglucosidase alfa provides patients with an exogenous form of GAA in the form of recombinant human (rh) GAA produced in transfected Chinese hamster ovary cells. In a clinical trial of 18 nonventilator-dependent infants treated with alglucosidase alfa beginning at or before 6 months of age, ${ }^{3}$ Cox regression analysis showed that treatment with alglucosidase alfa reduced the risk of death by $99 \%$ and reduced the risk of death or invasive ventilation by $92 \%$ at 18 months of age (both $P<0.0001$ ) relative to outcomes in an untreated historic reference cohort. In addition, left ventricular mass was reduced and a subgroup of patients achieved significant gross motor milestones. ${ }^{3}$

Although results from that study were significant, the study population was limited to young infants at the most severe end of the disease spectrum who were at a relatively early stage of disease progression when alglucosidase alfa treatment was initiated. We sought to evaluate whether treatment was safe and effective in patients with Pompe disease when alglucosidase

Priya S. Kishnani, MD, Department of Pediatrics, Division of Medical Genetics, Duke University Medical Center, Durham, NC 27710. E-mail: kishn001@mc.duke.edu.

Disclosure: P.S. Kishnani, M. Nicolino, and B. Byrne are members of the Pompe Disease Advisory Board for Genzyme Corporation. D. Bali and P. Kishnani have served as consultants for Genzyme Corporation. The clinical trials of Myozyme were supported by grants from Genzyme Corporation at the various sites at which patients were treated. Duke University and inventors of the method of treatment and predecessors of the cell lines used to generate the enzyme used in this clinical trial will benefit financially pursuant to Duke University's Policy on Inventions, Patents, and Technology Transfer, even if those cell lines are not used in the commercialized therapy.

Submitted for publication January 4, 2008

Accepted for publication October 13, 2008.

DOI: $10.1097 /$ GIM.0b013e31819d0996 
alfa therapy was initiated after 6 months of age. The present study was an open-label, multicenter, multinational evaluation of the safety and efficacy of alglucosidase alfa in a heterogeneous population of Pompe patients with onset of symptoms in infancy and evidence of cardiomyopathy who were at variable stages of disease progression (ranging from very advanced and near terminal to less advanced but still with significant effects in a variety of organ systems) when treatment was initiated.

\section{MATERIALS AND METHODS}

\section{Study design and treatment}

Key inclusion criteria were documented onset of Pompe disease symptoms by 12 months of age (corrected for gestation if born before 40 weeks); skin fibroblast GAA activity $\leq 2 \%$ of the normal mean; age 6-36 months at enrollment; and abnormal left ventricular mass indices (LVMIs, abnormal value is defined as $\geq 65 \mathrm{~g} / \mathrm{m}^{2}$ for patients up to 12 months old or $>79 \mathrm{~g} / \mathrm{m}^{2}$ for patients older than 12 months). ${ }^{4}$ Patients could be dependent on ventilator support or ventilator-free at enrollment. Exclusion criteria included clinical signs or symptoms of cardiac failure with ejection fraction $<40 \%$, major congenital anomaly, intercurrent organic disease, and prior treatment with ERT.

Patients were treated with IV alglucosidase alfa (Myozyme ${ }^{\circledR}$, provided by Genzyme Corporation) biweekly for a minimum of 52 weeks. Patients' guardians could then choose to continue treatment under the study protocol. All patients initially received a dose of 20 $\mathrm{mg} / \mathrm{kg}$ every 2 weeks. After at least 26 weeks of treatment, dose augmentation to $40 \mathrm{mg} / \mathrm{kg}$ every 2 weeks was allowed if the patient's clinical condition (motor, cardiac, or respiratory) had significantly deteriorated relative to baseline.

Independent Ethics Committees or Institutional Review Boards at sites in the United States, Europe, and Israel approved protocols and consent forms. Parents or guardians gave written informed consent for patients' participation. Independent boards oversaw safety (Data Safety Monitoring Board) and provided consultation on allergic reactions (Allergic Reaction Review Board). The study is registered at www.clinicaltrials.gov as NCT00053573.

\section{Historical control group}

Because infantile-onset Pompe disease is a rapidly fatal disorder and clinical trials have shown that treatment with rhGAA can improve survival, cardiac and respiratory function, growth, and motor development, ${ }^{5-9}$ ethics forbade using a placebo group in this study. Instead an untreated historic reference cohort was culled from a group of cases with infantile-onset Pompe disease identified through a retrospective chart review by Kishnani et al. ${ }^{10}$ The source group included 168 patients from nine countries and 33 different sites; $55 \%$ of patients were born in 1995 or later. The only inclusion criteria for this population were documented GAA enzyme deficiency or GAA gene mutation(s) and onset of signs or symptoms by 12 months of age. This group was screened with additional inclusion and exclusion criteria to closely resemble the clinical characteristics of the treated population in the current study.

Because patients with infantile-onset Pompe disease have a higher risk of death during the first 12 months of life than afterward, ${ }^{10}$ survival was analyzed separately for those patients who were $\leq 12$ months of age at first infusion, those patients who were $>12$ months of age, and all treated patients. Because of limited availability of detailed data regarding other clinical outcomes in the untreated reference cohort, only survival endpoints were compared with the reference cohort. For all other clinical endpoints, changes in the treated group were compared with baseline.

\section{Efficacy and safety}

The primary efficacy endpoint was the proportion of patients surviving over the course of treatment. ${ }^{11}$ Efficacy was further evaluated by measuring invasive and noninvasive ventilator dependency, LVMI, shortening fraction (an alternative to the ejection fraction for describing systolic cardiac function), growth, motor development, functional independence, GAA activity, and glycogen content in quadriceps muscle tissue.

The Alberta Infant Motor Scale, ${ }^{12}$ the Peabody Developmental Motor Scale, version 2 (PDMS-2), ${ }^{13}$ and the Pompe Pediatric Evaluation of Disability Inventory (PEDI), a disease-specific version of the PEDI, ${ }^{14-16}$ were used to assess motor development and functional independence at baseline and at regular intervals thereafter. Motor and cognitive assessments were scored by a central physical therapist. Although not an efficacy endpoint, cognitive function was assessed using the Bayley Scales of Infant Development, 2nd Edition (BSID-II). ${ }^{17}$

Left ventricular mass and shortening fraction were measured by echocardiography. Echocardiograms were read by a central cardiologist who was blinded to patient and time point through Week 52 .

To assess safety, patients were observed and vital signs recorded during each infusion and for 2 hours afterward. Adverse events, including infusion-associated reactions (IARs), were continuously monitored in all patients.

Anti-rhGAA IgG antibody formation was assessed with an enzyme-linked immunosorbent assay and confirmed with a radioimmunoprecipitation assay as previously described. ${ }^{9}$ Enzyme activity and flow cytometry-based assays were performed retrospectively in all seroconverted patients to detect inhibitory antibodies in patient frozen serum. These two assays were used to characterize antibody response by inhibition of rhGAA enzyme activity and interference with uptake into fibroblasts, respectively.

GAA activity in skin fibroblasts (at baseline) and quadriceps muscle (at baseline and Weeks 12 and 52) was assayed using methods previously described. ${ }^{9}$ Cross-reactive immunologic material (CRIM) status was determined as described previously. ${ }^{9}$ Briefly, cell lysates derived from patients' fibroblasts were subjected to western blot analysis with a pool of monoclonal antibodies that recognize both native and recombinant GAA; results were confirmed independently by a second laboratory that performed western blot on blinded cell lysate samples using polyclonal antibodies generated against human placental GAA.

\section{Statistical methods}

To include all available follow-up data in the analyses, data were analyzed at various time points, including 52 weeks, 104 weeks, last available follow-up (the last time point at which a given outcome was measured), and end of study. Survival over time and ventilation-free survival were analyzed using KaplanMeier time-to-event analysis ${ }^{11}$; the binomial proportions of patients alive and ventilation-free at the end of study were also calculated. A Cox proportional-hazards model ${ }^{18}$ incorporating age at diagnosis and age at symptom onset, with treatment duration as a time-varying covariate, was used to compare survival and ventilation-free survival in the study patients to survival in the untreated historical reference cohort.

\section{RESULTS}

\section{Patient population}

Twenty-two patients were enrolled but only 21 were treated, as one patient died of anesthesia-related complications during a procedure to obtain a muscle biopsy before beginning algluco- 
sidase alfa treatment. Four age-related protocol deviations were agreed to by the sponsor and the treating physician: three patients were older than 36 months of age (36.6, 37.3, and 43.1 months) and one was 3.7 months of age at the time of first infusion. The older patients all showed significant fine and gross motor development delays, were not able to stand or walk independently, and had LVMI $Z$ scores of at least 5.4; the 3.7-month-old patient already required ventilator support, and so was ineligible for other clinical studies of alglucosidase alfa.

The population was $48 \%$ male and predominantly white (Table 1). Baseline GAA activity measured in the skin fibroblasts was negligible $(<1 \%)$ for all patients; two patients were CRIM negative. The median age at first infusion was 13 months (Table 1). All but one infused patient presented with symptoms by 12 months of age (median 3.0 months; range, $0.0-12.6$; ages not corrected for gestational age at birth). One patient was included whose first symptoms were recorded at age 12.6 months, or 12.1 month when corrected for gestational age at birth; although this patient did not meet the age at symptom onset criterion, the deviation was felt to be quite small and unlikely to affect the study outcome. GAA gene mutations were analyzed in all patients who consented $(n=18)$; each patient had a unique combination of mutations (data not shown) and none had the IVS1 (c. $-32-13 \mathrm{~T} \rightarrow \mathrm{G}$ ) mutation that is the most common mutation in adult Pompe patients. ${ }^{19}$

Of the 168 patients in the source population for the untreated historic reference cohort, 84 met the inclusion and exclusion criteria and had survival data; these were used as comparators in the survival analyses. Demographics and baseline characteristics were comparable with the study population (data not shown).

\section{Efficacy results}

\section{Survival and ventilator use}

Fifteen of 21 patients $(71 \%)$ treated with alglucosidase alfa for a median of 120 weeks (range, 0.6-168 weeks) were alive at the end of the study period. The six patients who died ranged

Table 1 Patient demographics and baseline characteristics

\begin{tabular}{|c|c|c|}
\hline & Study population & Untreated reference cohort (for survival analysis only) \\
\hline Parameter & $n=21$ & $n=86$ \\
\hline \multicolumn{3}{|l|}{ Gender, $n(\%)$} \\
\hline Male & $10(48 \%)$ & $36(42 \%)$ \\
\hline Female & $11(52 \%)$ & $50(58 \%)$ \\
\hline \multicolumn{3}{|l|}{ Race, $n(\%)$} \\
\hline White & $15(71 \%)$ & $40(47 \%)$ \\
\hline Black & $2(10 \%)$ & $12(14 \%)$ \\
\hline Hispanic & 0 & $1(1 \%)$ \\
\hline Asian & $3(14 \%)$ & $28(33 \%)$ \\
\hline Other/unknown & $1(5 \%)$ & $5(6 \%)$ \\
\hline \multicolumn{3}{|l|}{ Age at first symptoms, mo ${ }^{a}$} \\
\hline Mean $\pm \mathrm{SD}$ & $3.9 \pm 2.8$ & $3.1 \pm 2.75$ \\
\hline Median (min, max) & $3.0(0.0,12.6)$ & $3.0(0.0,12.0)$ \\
\hline \multicolumn{3}{|l|}{ Age at diagnosis, mo } \\
\hline Mean $\pm \mathrm{SD}$ & $8.8 \pm 5.4$ & $5.8 \pm 3.81$ \\
\hline Median (min, max) & $6.8(1.5,22.6)$ & $5.7(-5.1,22.7)$ \\
\hline \multicolumn{3}{|l|}{ Age at first infusion, $\mathrm{mo}^{a}$} \\
\hline Mean \pm SD & $15.7 \pm 11.0$ & \\
\hline Median (min, max) & $13.0(3.7,43.1)$ & \\
\hline \multicolumn{3}{|c|}{ Age at death in historical reference cohort, $\mathrm{mo}^{a}$} \\
\hline Median (min, max) & & $9.8(5.9,47.9)$ \\
\hline $95 \% \mathrm{CI}$ & & $8.6-10.8$ \\
\hline \multicolumn{3}{|c|}{ Ventilator support at baseline (first infusion) } \\
\hline Invasive ventilation & $5(24 \%)$ & \\
\hline Noninvasive ventilation & $2(10 \%)$ & \\
\hline No ventilator support & $14(67 \%)$ & \\
\hline
\end{tabular}


in age from 3.7 to 13.0 months at first infusion and from 7.7 to 27.1 months at time of death. Five of these patients died before 18 months of age and early in the course of treatment (before Week 28); the sixth patient survived until Week 101. The patients who survived ranged in age from 7.0 to 43.1 months (mean, 18.8; median, 16.2) at first infusion and from 34.7 to 80.3 months at study end. None of the deaths were assessed by the treating physician to be related to treatment; causes of death were cardiac and/or respiratory failure in all cases.

Before treatment, of the 21 patients 16 were free of invasive ventilation (two of those 16 patients were noninvasively ventilated, via mask) and five required 24-hour invasive ventilation (via tracheostomy). At the end of the study period, seven of these 16 patients remained alive and free of invasive ventilation, four had become invasive ventilation-dependent, and five had died. Among the five patients who were invasively ventilated at baseline, three continued to require invasive ventilation 24 hours a day, one decreased the number of hours of ventilation from 24 hours to 12 hours, and one died (Table 2).

The Kaplan-Meier survival estimate at 104 weeks, the last evaluable time point, was $71.1 \%$ (95\% [confidence interval] CI: $51.6-90.6 \%$ ) for study patients, compared with $26.3 \%$ (95\% CI: $6.5-46.1 \%$ ) in the reference group (Table 3 ). When patients were divided by age at first infusion, there was no overlap between the $95 \%$ CIs for the 104-week survival estimates between the study group and the reference group. Cox regression analysis ${ }^{18}$ comparing treated patients with patients in the historical reference group indicated that treatment with alglucosidase alfa reduced the risk of death by $79 \%(P=0.0009)$ and the risk of death or invasive ventilation by $58 \%(P=0.0207)$ over the course of the study (Table 4). Note that the risk of death or invasive ventilation in the study group was compared with risk of death only in the historical reference group, because reliable information on ventilation status was not available. Risk reduction for death or any type of ventilation was not statistically significant.

\section{Cardiac response}

At baseline, all patients showed evidence of left ventricular hypertrophy (abnormal LVMI and LVM $Z$ score $>2$ ) as assessed by the enrolling sites. This assessment was confirmed by a central, blinded cardiologist who concurred with the sites in 18 of 19 cases where echocardiograms were of sufficient quality to allow reassessment; one patient had a baseline LVM $Z$ score of 1.7 on reassessment. Over the course of the study, mean LVMI improved steadily, resulting in a $42 \%$ reduction by Week 52 and a $63 \%$ reduction by Week 104 (Table 5). Beyond Week 12 , all patients with echocardiograms who had elevated LVMI experienced further decreases in LVMI and those who were within the normal range maintained normal LV mass. By the time of last echocardiography assessment, $81 \%$ (17/21) of patients showed a reduction in LVM, including 12 patients who achieved normal LVM $(Z$ score $<2$; Fig. 1$)$. Of the four patients in whom LVM did not decrease, one died before any repeat assessment, and the other three died before the Week 24 assessment. Mean shortening fraction (SF) $Z$ score at baseline was -1.2. After an initial drop, SF also improved over time (Fig. 1, Table 5), with mean SF increasing over baseline by $22 \%$ at Week 52 and $30 \%$ at Week 104.

Baseline and follow-up LVM $Z$ scores were also analyzed for patients according to age at first infusion. Mean LVM $Z$ score at baseline was $6.7 \pm 1.64$ for patients $\leq 12$ months of age at first infusion $(n=9)$ and $4.2 \pm 2.45$ for patients $>12$ months of age at first infusion $(n=10)$, suggesting that younger patients had more rapid disease progression. At 52 weeks, only four patients in the younger group had evaluable echocardiograms; for these patients mean LVM $Z$ score had decreased to $2.5 \pm 3.5$. The mean LVM $Z$ score at 52 weeks for patients $>12$ months of age at first infusion $(n=9)$ was $1.6 \pm 1.91$. No evaluation of statistical significance for the change over time between the two groups was attempted because of the small sample size.

\section{Muscle GAA activity and glycogen content}

Mean GAA in muscle at baseline was $12.5 \pm 11.0 \mathrm{nmol} /$ hour/g. By Week 52, all patients receiving repeat biopsies $(n=$ 13) experienced a marked increase in GAA activity with mean levels of $89.9 \pm 32.2 \mathrm{nmol} /$ hour $/ \mathrm{g}$. Mean glycogen value in muscle by biochemical analysis at baseline was $7.1 \pm 2.5 \%$. After 52 weeks of treatment, 13 patients had repeat biopsies assessed: glycogen content decreased for six patients, remained stable for three patients, and increased for four patients relative to baseline, and mean glycogen decreased to $5.0 \pm 2.4 \%$ (Table 2).

\section{Motor development}

Thirteen of 21 patients $(62 \%)$ had measurable motor development gains (AIMS and/or PDMS-2 gross and fine motor skills) as determined by increases in age-equivalent scores from baseline to end of study. Of the 13 patients with significant motor gains, five patients had functional use of the legs and were walking independently and eight patients had functional use of the neck, trunk, and upper extremities and were sitting at the end of the study (Table 2). The remaining eight patients (38\%) made no significant motor development gains from baseline. At baseline, these eight patients had median muscle GAA activity below quantifiable levels; they also had generally lower motor scores at baseline (data not shown). The 13 patients who had measureable motor development gains had higher baseline median muscle GAA activity $(15.6 \mathrm{nmol} / \mathrm{hour} / \mathrm{g}$ tissue versus below quantifiable levels) and better motor scores at baseline (data not shown). Five of these 13 patients were younger than 12 months old at the time of their first infusion, and five of the seven patients who did not have measurable motor gains were also under 12 months of age at first infusion (Table 2).

\section{Functional independence skills}

Pompe PEDI scores steadily increased over the course of the study indicating the acquisition of functional skills. From baseline to last assessment, 17 of 21 patients $(81 \%)$ demonstrated consistent gains in at least one of the domains assessed, indicating increased ability to independently perform activities of daily living related to mobility, self-care, and/or social function. Three of the four patients without gains died before Week 24 assessment.

Two of 11 patients who were older than 12 months at time of first infusion showed no improvement in Pompe PEDI scores. Of those nine who did improve, six showed measurable improvement in all three domains assessed. Of the 10 patients younger than 12 months at first infusion, three showed improvements in all three Pompe PEDI domains.

\section{Physical growth}

Growth in body weight and length generally progressed over the course of the study (Fig. 2). By last assessment, ageequivalent weight values were at or above the third percentile in $81 \%(17 / 21)$ of patients. Results for age-equivalent length were similar, with $90 \%(19 / 21)$ of patients at or above the third percentile at last assessment. Two patients started below the third percentile in weight, did not improve, and died with $<16$ weeks on therapy; one patient declined in age-equivalent weight and subsequently died after 28 weeks on therapy. One patient 


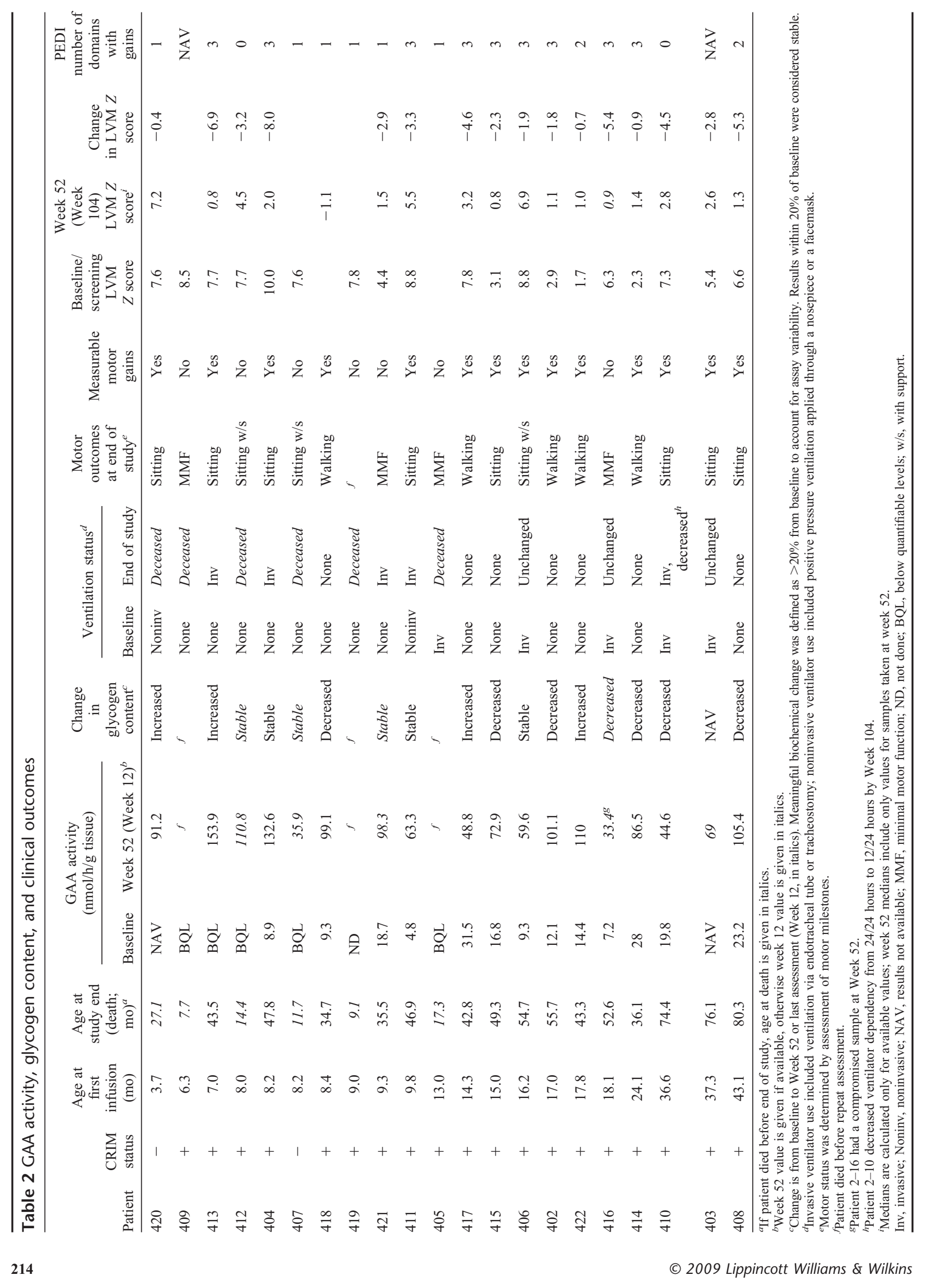


Table 3 Survival estimates for patients aged $\leq 12 \mathrm{mo}$ and $>12 \mathrm{mo}$ at first infusion compared with survival in the untreated reference cohort

\begin{tabular}{|c|c|c|c|c|c|}
\hline \multicolumn{4}{|c|}{ Study patients } & \multicolumn{2}{|r|}{ Untreated reference cohort } \\
\hline Age category $(\mathrm{mo})^{a}$ & $N$ & $\begin{array}{l}\text { Median age at first infusion } \\
\text { (mo) }\end{array}$ & 104-week survival estimate ${ }^{b}$ & $N$ & $\begin{array}{c}\text { Conditional 104-week survival } \\
\text { estimate }{ }^{b, c}\end{array}$ \\
\hline$\leq 12$ & 10 & 8.2 & $50.0 \%(19.0 \%, 81.0 \%)$ & 59 & $9.2 \%(1.5 \%, 16.8 \%)$ \\
\hline$>12$ & 11 & 17.8 & $90.9 \%(73.9 \%, 100.0 \%)$ & 11 & $45.5 \%(16.0 \%, 74.9 \%)$ \\
\hline All patients & 21 & 13.0 & $71.1 \%(51.6 \%, 90.6 \%)$ & 19 & $26.3 \%(6.5 \%, 46.1 \%)$ \\
\hline
\end{tabular}

${ }^{a}$ Age category is age at first infusion for study patients and age at death for the reference cohort.

${ }^{b}$ The Kaplan-Meier method was used to compute nonparametric estimates of the survival distribution. Values are estimate and $95 \%$ CI.

${ }^{c}$ Conditional survival estimates in the reference cohort were based on median age at first infusion for the corresponding study subpopulation (e.g., 104-week conditional survival rate corresponded to survival rate at $8.2+24=32.2$ mo of age for the $\leq 12$-mo category).

Table 4 Survival and ventilator-free survival in patients treated with alglucosidase alfa compared with the untreated reference cohort $(N=21$, treated patients; $N=84$, reference cohort)

\begin{tabular}{lccrr}
\hline End point & Treatment effect hazard ratio & 95\% confidence interval & $P$ & Risk reduction \\
\hline Survival & 0.209 & $0.083-0.524$ & 0.0009 & $79 \%$ \\
Invasive ventilator-free survival & 0.421 & $0.202-0.876$ & 0.0207 & $58 \%$ \\
Any ventilator-free survival & 0.533 & $0.247-1.150$ & 0.1088 & $47 \%$ \\
\hline
\end{tabular}

Results are from a Cox proportional hazards regression analysis which includes treatment as a time-varying covariate, and also includes age of diagnosis and age at symptom onset. Analysis is from time of diagnosis through the end of study.

Table 5 Cardiac status by echocardiography

\begin{tabular}{|c|c|c|c|c|c|c|c|c|}
\hline \multirow[b]{2}{*}{ Measure } & \multicolumn{2}{|c|}{ Baseline } & \multicolumn{2}{|c|}{ Week 52} & \multicolumn{2}{|c|}{ Week 104} & \multicolumn{2}{|c|}{$\begin{array}{l}\text { Mean change from } \\
\text { baseline to week } 104\end{array}$} \\
\hline & $n$ & Mean $\pm \mathrm{SD}$ & $n$ & Mean \pm SD & $n$ & Mean \pm SD & $n$ & $\%$ Change \\
\hline LVMI & 19 & $193.8 \pm 98.5$ & 13 & $89.3 \pm 58.8$ & 10 & $53.1 \pm 13.9$ & 9 & -62.7 \\
\hline LVM $Z$ score & 19 & $6.5 \pm 2.6$ & 13 & $2.7 \pm 2.5$ & 10 & $0.9 \pm 0.8$ & 11 & -5.3 \\
\hline Shortening fraction & 21 & $35.6 \pm 12.1$ & 13 & $45.5 \pm 5.6$ & 10 & $43.8 \pm 6.5$ & 10 & 30.4 \\
\hline
\end{tabular}

started below the third percentile in height and remained at a consistent height percentile throughout the study; this patient's age-equivalent weight increased from percentile 1.3 to 7.7 over the same time period. One patient died before any repeat growth assessment.

\section{Cognitive function}

Eighteen of 19 patients with follow-up data showed improvements in age-equivalent BSID-II scores from baseline to last assessment. The one patient who failed to demonstrate consistent improvement in BSID-II (and Pompe PEDI) scores to study end also experienced significant motor function loss after 1 year of therapy.

\section{Dose augmentation}

Eight patients received $40 \mathrm{mg} / \mathrm{kg}$ dose after a minimum of 26 weeks on alglucosidase alfa. Of these, five qualified for dose increase because of failure to demonstrate a clinically meaningful change from baseline in the Functional Skills Mobility domain score of the Pompe PEDI; four patients qualified because they required new respiratory support or had a clinically meaningful increase in the amount of required respiratory support (two of these also qualified based on motor function); and one patient qualified because of the development of clinical symptoms of cardiac failure. Clinical status for these patients remained largely unchanged after the dose increase; one patient resolved symptoms of cardiac failure but subsequently died of respiratory failure.

\section{Safety results}

\section{Adverse events/infusion-associated events}

Eleven (52\%) patients experienced 42 IARs, which were defined as AEs occurring on the day of infusion and assessed by the investigator as being related to treatment. The most common IARs were skin and subcutaneous skin disorders (13 events); vascular disorders (10 events); and oxygen saturation, blood pressure increase, heart rate increase or respiratory rate increase (seven events). Flushing occurred in four patients, oxygen desaturation in three patients, and urticaria, pyrexia, cough, and tachypnea were observed in two patients each. Reactions were managed by slowing the infusion rate or temporarily stopping 

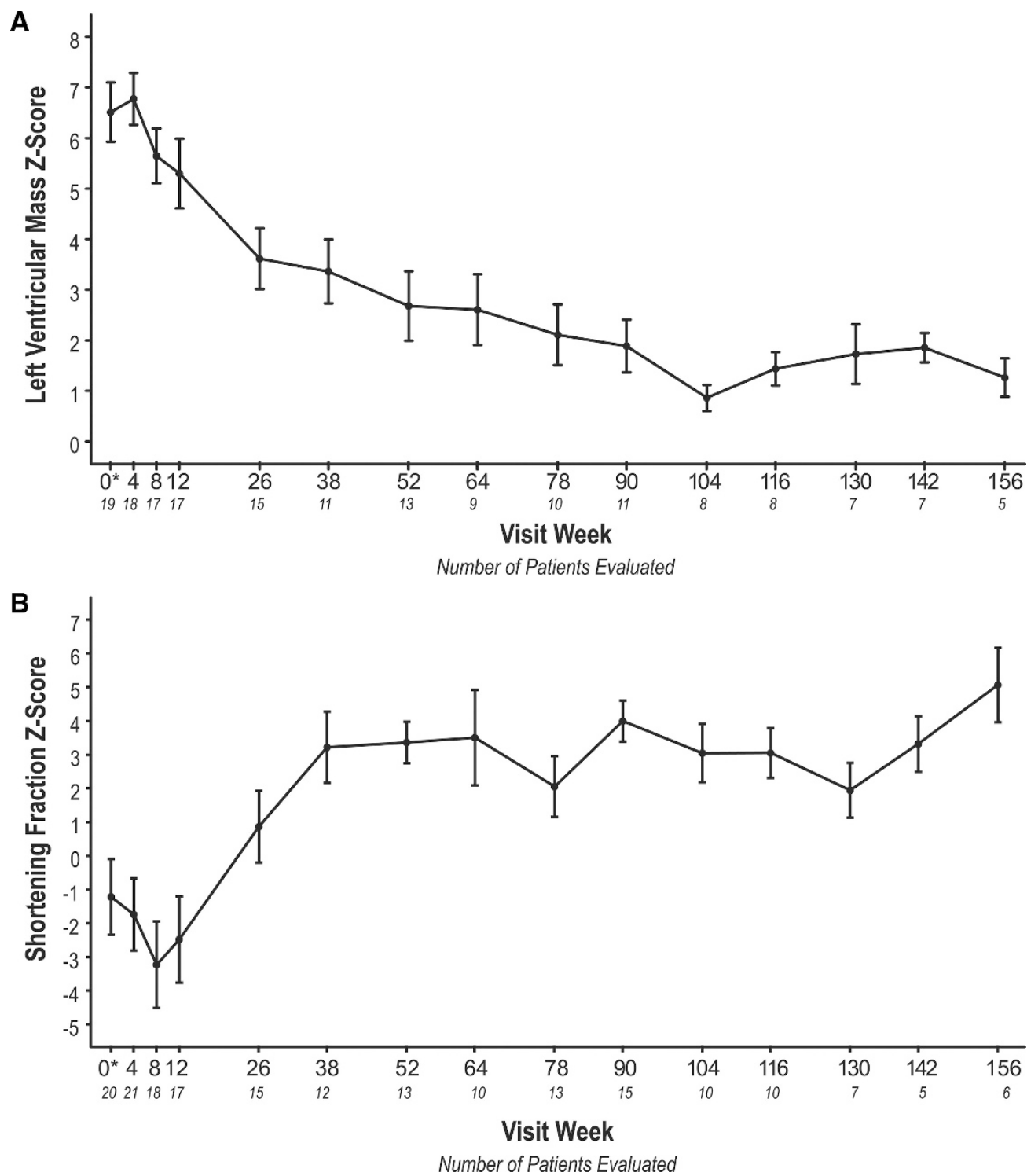

Fig. 1. (A) Mean LVM Z scores and (B) mean shortening fraction $Z$ scores from baseline to last assessment; vertical bars indicate standard error. Dotted line represents a normal $Z$ score.

the infusion and administering symptomatic treatment. All 11 patients recovered without sequelae and continued treatment. Retrospective analysis of two patients with IARs suggestive of hypersensitivity reactions found those patients to be IgE negative. Infusion interruption and symptomatic treatment resolved these reactions and both patients were rechallenged successfully.

Six patients died during the treatment period; none of the deaths were attributed to treatment. All of the deaths were attributed to cardiac and/or respiratory causes, including cardiac or cardiorespiratory arrest, acute pulmonary edema, arrhythmia, and respiratory failure secondary to bronchiolitis.

\section{Antibody response}

Nineteen of $20(95 \%)$ patients with postbaseline blood draws developed IgG antibodies to alglucosidase alfa. All but one of these patients seroconverted by Week 12; the median time to seroconversion was 4 weeks. The one patient who remained seronegative died before Week 8 . Of the 19 patients who seroconverted, two tested negative for IgG antibodies at their last assessment (at Weeks 104 and 156); the maximum titer reported for both of these patients was 400. At last assessment, 14 patients had antibody titers between 200 and 6400, whereas three patients had titers between 51,200 and 102,400. Of note, last IgG antibody titers in the two CRIM-patients were 102,400 (patient died in Week 15) and 6,400 (patient died in Week 101). None of the patients who seroconverted exhibited significant inhibitory antibody activity by either assay at any time point.

\section{DISCUSSION}

In this study, a group of infants and children with Pompe disease treated with alglucosidase alfa demonstrated improved survival and invasive ventilation-free survival in comparison 

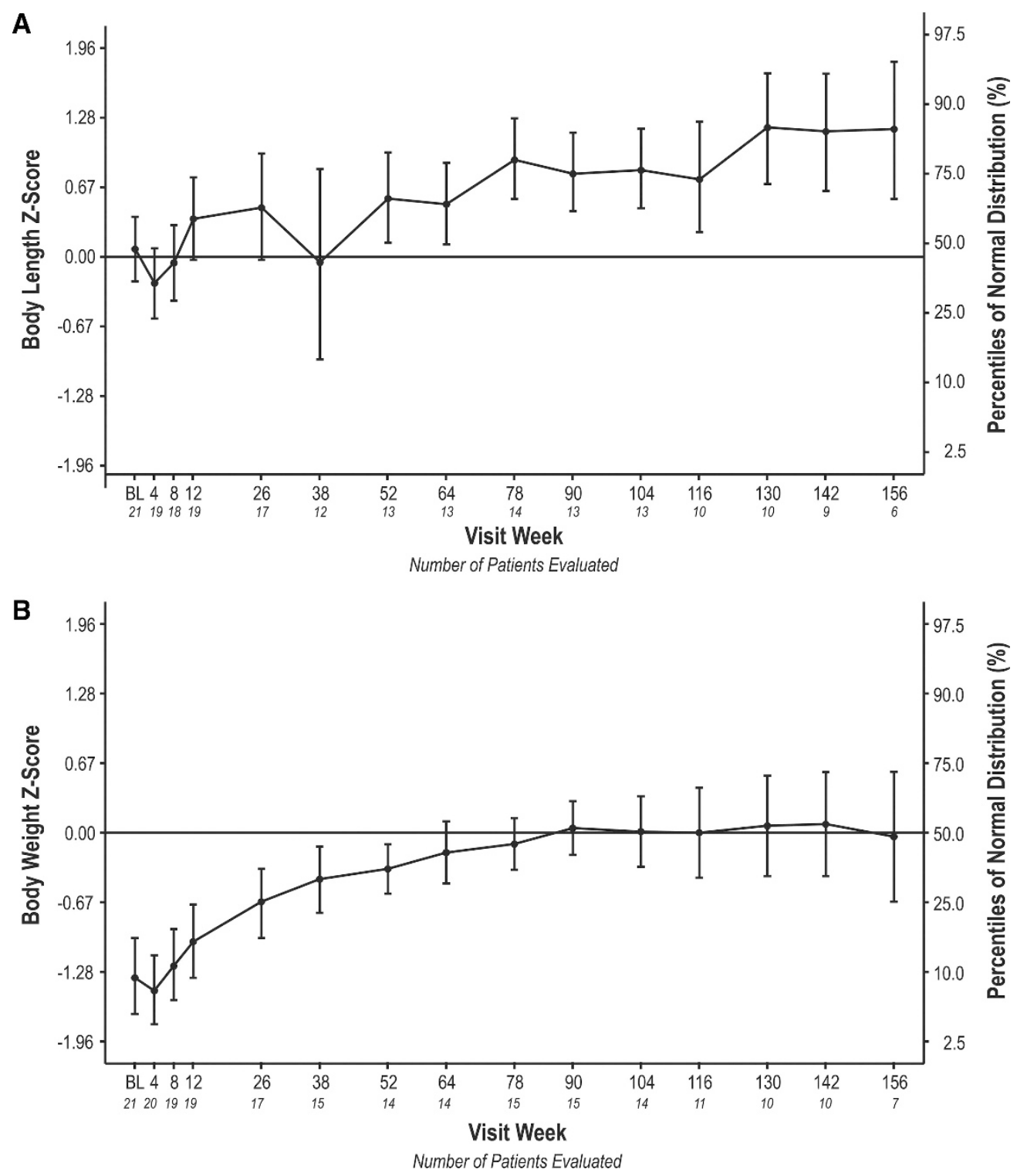

Fig. 2. (A) Mean body length $Z$ scores and (B) mean body weight $Z$ scores and relation to CDC growth chart norms.

with patients in an untreated reference cohort. All but one patient had onset of symptoms in the first 12 months of life and all exhibited signs of advanced disease (e.g., need for ventilator support, markedly delayed motor development, and/or elevated LVMI). A previous study showed that alglucosidase alfa significantly extended survival and ventilator-free survival in a more homogeneous group of young infants who were treated at a relatively early stage of disease progression. ${ }^{3}$ The present study shows that this benefit extends to older infants and children.

In addition to improving survival in young patients, alglucosidase alfa has also been shown to improve Pompe disease manifestations such as indices of cardiomyopathy, and to allow patients to continue motor development beyond the point at which it is arrested in untreated patients. ${ }^{3,9}$ In the present study, clear improvements over baseline were demonstrated in these areas. Although these outcomes were not directly compared with the untreated cohort, they contrast sharply with the well- documented natural course of the disease in infants and young children. ${ }^{10,20}$

In any rapidly progressing disease, age is typically associated with worsening clinical status. In natural history studies of Pompe disease, patients presenting with symptoms in the first year of life have poor prognoses. The historical, rigid separation of Pompe disease into infantile-onset and late-onset has come into question recently as more is learned about the inconsistency of age at clinical presentation and progression of disease; age at onset of symptoms of disease does not always correspond with speed of progression or prognosis. The current cohort is a group of patients with rapid yet variable disease progression, as demonstrated by the heterogeneity of age and clinical involvement at initiation of treatment. We included only patients with onset of disease symptoms in infancy and, with one exception, measurable cardiac involvement. Although these are the generally accepted criteria for infantile-onset Pompe disease, it is clear that the rate of disease progression varied in this cohort. Previ- 
ous studies indicate that where there is cardiac involvement and motor impairment, disease progression is generally rapid in the absence of treatment. In this study, response to treatment was generally more pronounced in patients with better preservation of cardiac, respiratory, or skeletal muscle function at baseline. It is notable that patients who were older at the time of first ERT infusion (and therefore likely had a more slowly progressive disease course) had a better response to treatment. Of the four oldest patients, two normalized their LVM $Z$ scores and all four had measurable motor gains with treatment. However, despite their advanced age by the end of the study (3-6 years, with treatment duration between 12 and 37 months), only one of these patients could walk by the end of the study and two remained dependent on invasive ventilation. These results suggest that baseline clinical status is a better indicator of Pompe disease progression and potential response to treatment than age alone.

In untreated Pompe disease, cardiomyopathy parameters observed in infants worsen over time and eventually lead to congestive heart failure. ${ }^{10,20}$ In this study, all patients with echocardiograms beyond 12 weeks demonstrated improvement or maintenance of normal LVMI; notably more than half of patients attained a normal LVM $(Z$ score $\leq 2)$ by last assessment. Measurements of cardiac function also improved; the initial, transient drop in mean SF that was observed may be due to an initial remodeling phase potentially prompted by the rapid removal of glycogen from the cardiac walls. The overall improvement in cardiac status likely contributed to the prolonged survival rates observed in this study.

Motor development is often completely arrested in untreated infants and children with Pompe disease, or if motor milestones are achieved, they are subsequently lost. ${ }^{10,20}$ In the present study, many patients acquired new motor and functional skills. The majority of patients who did not show motor gains had undetectable muscle GAA activity and more advanced disease at baseline, including lower age-equivalent motor scores. Additionally, almost all patients in the study demonstrated the acquisition of functional skills as measured by the Pompe PEDI; this measure provides a more sensitive and comprehensive assessment of the ability to perform skills required for many activities of daily living than motor evaluations alone. ${ }^{15,16}$

Failure to thrive is another typical finding in infants with untreated Pompe disease. ${ }^{10,20}$ A natural history study found that, despite the frequent use of tube feeding (in 59.5\% of patients), $53 \%$ of untreated Pompe patients failed to maintain normal growth in the first year of life. ${ }^{10}$ In contrast, in the present study, almost all patients maintained weight- and length-for-age percentiles above the 3 rd percentile through the study. It should be noted that patients who required long-term invasive ventilation at any point in the study received tube feedings.

BSID-2 age-equivalent scores indicated continued cognitive, language, and personal-social development for the majority of patients. Little is known about cognitive development in infants with Pompe disease because of the early mortality observed in untreated patients; the interpretation of the findings observed is complicated by the difficulties inherent in performing a standardized test administration to patients who have oral-motor and gross- and fine-motor deficits, as well as possible hearing loss,$^{21}$ which may significantly affect test performance.

Previously published data suggest that the extent of muscle damage may predict response to treatment with alglucosidase alfa. ${ }^{9,22}$ The results of this study provide additional support for this observation. Four patients who died before Week 52 had negligible baseline muscle GAA activity levels. Motor function at baseline was also worse in these patients (four had age- equivalent baseline motor scores of 1 month or lower) and one was already invasively ventilated at baseline. The patients who were walking or sitting independently at the end of the study, in contrast, had higher muscle GAA activity and better baseline motor function at baseline than those who made no significant motor gains, suggesting greater preservation of muscle tissue.

Although alglucosidase alfa seems to have increased the activity of GAA in muscle in this study, GAA activity levels changes did not consistently correlate with muscle glycogen content. This result is consistent with previous findings that increased muscle GAA activity after ERT does not always correlate with clearing glycogen from muscle tissues or with motor improvements. ${ }^{9,10}$ It is notable that samples in this and previous studies ${ }^{9,10}$ were taken exclusively from quadriceps; glycogen content measurements may not be consistent across various muscle groups in a single patient. Animal model studies have shown that glycogen clearance after rhGAA administration decreased as glycogen load increased (as animals aged). ${ }^{23}$ Different muscle fiber types with varying density of receptors and degrees of autophagy may exhibit differential clearance of glycogen in the presence of enhanced GAA activity. ${ }^{23-25}$

Although approximately half of the patients treated with alglucosidase alfa in this study experienced IARs, none of the IARs led to the cessation of treatment. Although most patients developed antibodies against alglucosidase alfa, the majority showed a trend toward decreasing titers over time and none had evidence of in vitro inhibitory activity. The higher $(40 \mathrm{mg} / \mathrm{kg})$ dose of alglucosidase alfa was generally well tolerated, but this dose did not seem to change patients' clinical response. However, assessments and analyses were not resynchronized after a patient changed doses, and therefore meaningful conclusions regarding the safety or efficacy of the higher dose cannot be drawn from this study.

\section{CONCLUSION}

In this heterogeneous population of infants and children with advanced Pompe disease, ERT with alglucosidase alfa beginning after 6 months of age extended survival and invasive ventilator-free survival compared with the untreated reference group. In addition, the majority of patients treated with alglucosidase alfa improved echocardiographic indices of cardiomyopathy and made growth and motor development gains not typically observed in untreated infants and children. The results of this study are promising in that they show that alglucosidase alfa can reduce the mortality caused by this rapidly progressive, life-threatening disorder even when treatment is started at a more advanced stage of disease.

\section{ACKNOWLEDGMENTS}

This study was sponsored by Genzyme Corporation. The authors thank the patients who participated in this clinical study and their families. They also thank the treating physicians, study coordinators, nurses, and technical staff at the various treatment centers. Drs. Jamie Levine and Carolyn Spencer provided crucial guidance in study design as well as central evaluation services. They thank Y. T. Chen, MD, for his review of the manuscript. They acknowledge Michael Friedman, PhD, for assistance with statistical analyses, and Katherine Lewis, MA, and Victoria DeMoranville, MA, for editorial assistance (all from Genzyme Corporation). 


\section{REFERENCES}

1. Hirschhorn R, editor. The metabolic and molecular basis of inherited diseases, 8th ed. New York: Mcgraw-Hill, 2001.

2. Kishnani PS, Howell RR. Pompe disease in infants and children. $J$ Pediatr 2004;144(suppl 5):S35-S43.

3. Kishnani PS, Corzo D, Nicolino M, et al. Recombinant human acid [alpha]glucosidase: major clinical benefits in infantile-onset Pompe disease. Neurology 2007;68:99-109.

4. Vogel M, Staller W, Buhlmeyer K. Left ventricular myocardial mass determined by cross-sectional echocardiography in normal newborns, infants, and children. Pediatr Cardiol 1991;12:143-149.

5. Amalfitano A, Bengur AR, Morse RP, et al. Recombinant human acid alpha-glucosidase enzyme therapy for infantile glycogen storage disease type II: results of a phase I/II clinical trial. Genet Med 2001;3:132-138.

6. Van den Hout JM, Reuser AJ, de Klerk JB, Arts WF, Smeitink JA, Van der Ploeg AT. Enzyme therapy for pompe disease with recombinant human alpha-glucosidase from rabbit milk. J Inherit Metab Dis 2001;24:266274.

7. Van den Hout JM, Kamphoven JH, Winkel LP, et al. Long-term intravenous treatment of Pompe disease with recombinant human alpha-glucosidase from milk. Pediatrics 2004;113:e448-e457.

8. Klinge L, Straub V, Neudorf U, et al. Safety and efficacy of recombinant acid alpha-glucosidase (rhGAA) in patients with classical infantile Pompe disease: results of a phase II clinical trial. Neuromuscul Disord 2005;15:24-31.

9. Kishnani PS, Nicolino M, Voit T, et al. Chinese hamster ovary cell-derived recombinant human acid alpha-glucosidase in infantile-onset Pompe disease. J Pediatr 2006;149:89-97.

10. Kishnani PS, Hwu WL, Mandel H, et al. A retrospective, multinational, multicenter study on the natural history of infantile-onset Pompe disease. J Pediatr 2006;148:671-676.

11. Kaplan EL, Meier P. Nonparametric estimation from incomplete observations. J Am Stat Assoc 1958;53:457-481.

12. Piper M. Motor assessment of the developing infant. Philadelphia: Saunders, 1994.
13. Folio MR, Fewell RR. Peabody developmental motor scales, Second Edition (PDMS-2). Austin, TX: PRO-ED, Inc., 2000.

14. Feldman AB, Haley SM, Coryell J. Concurrent and construct validity of the Pediatric Evaluation of Disability Inventory. Phys Ther 1990;70:602-610.

15. Haley SM, Fragala MA, Aseltine R, Ni P, Skrinar AM. Development of a disease-specific disability instrument for Pompe disease. Pediatr Rehabil 2003;6:77-84.

16. Haley SM, Fragala-Pinkham MA, Ni PS, Skrinar AM, Kaye EM. Pediatric physical functioning reference curves. Pediatr Neurol 2004;31:333-341.

17. Bayley N. Bayley scales of infant development-second edition (BSID-II). San Antonio, TX: Psychological Corp., 1993.

18. Cox DR. Regression models and life tables. J Royal Stat Soc Ser B (Methodological) 1972;34:187-220.

19. Kroos MA, Pomponio RJ, Hagemans ML, et al. Broad spectrum of Pompe disease in patients with the same c.-32-13T- $>$ G haplotype. Neurology 2007; 68:110-115.

20. van den Hout HM, Hop W, van Diggelen OP, et al. The natural course of infantile Pompe's disease: 20 original cases compared with 133 cases from the literature. Pediatrics 2003;112:332-340.

21. Kamphoven JH, de Ruiter MM, Winkel LP, et al. Hearing loss in infantile Pompe's disease and determination of underlying pathology in the knockout mouse. Neurobiol Dis 2004;16:14-20.

22. van der Beek NA, Hagemans ML, van der Ploeg AT, Reuser AJ, van Doorn PA. Pompe disease (glycogen storage disease type II): clinical features and enzyme replacement therapy. Acta Neurol Belg 2006;106:82-86.

23. Raben N, Jatkar T, Lee A, Lu N, et al. Glycogen stored in skeletal but not in cardiac muscle in acid alpha-glucosidase mutant (Pompe) mice is highly resistant to transgene-encoded human enzyme. Mol Ther 2002;6:601-608.

24. Hawes ML, Kennedy W, O'Callaghan MW, Thurberg BL. Differential muscular glycogen clearance after enzyme replacement therapy in a mouse model of Pompe disease. Mol Genet Metab 2007;91:343-351.

25. Raben N, Danon M, Gilbert AL, Dwivedi S, et al. Enzyme replacement therapy in the mouse model of Pompe disease. Mol Genet Metab 2003;80:159-169. 